\title{
GENERALIZED HARTEN FORMALISM AND LONGITUDINAL VARIATION DIMINISHING SCHEMES FOR LINEAR ADVECTION ON ARBITRARY GRIDS*
}

\author{
Bruno Després ${ }^{1,2}$ AND FrÉdÉRIC LAGOUtière ${ }^{2}$
}

\begin{abstract}
We study a family of non linear schemes for the numerical solution of linear advection on arbitrary grids in several space dimension. A proof of weak convergence of the family of schemes is given, based on a new Longitudinal Variation Diminishing (LVD) estimate. This estimate is a multidimensional equivalent to the well-known TVD estimate in one dimension. The proof uses a corollary of the Perron-Frobenius theorem applied to a generalized Harten formalism.

Résumé. Nous étudions une famille de schémas non linéaires pour l'approximation numérique de l'advection linéaire sur grille quelconque en dimension d'espace supérieure à un. Une preuve de convergence est proposée à partir d'une estimation de la variation longitudinale. Cette estimation est une généralisation multidimensionnelle discrète de l'estimation TVD discrète, bien connue en dimension un d'espace.
\end{abstract}

Mathematics Subject Classification. 76M12, 65M12.

Received: April 26, 2001. Revised: July 19, 2001.

\section{INTRODUCTION}

We address the numerical solution of linear advection in several space dimensions on triangular or quadrangular arbitrary grids, with either linear or non linear schemes. This problem relies on the general theory of numerical approximation of scalar linear and non linear hyperbolic equations by means of finite volume methods. In his seminal work in 1D (in [21,22]), Harten introduced what is commonly referred to as the Harten formalism. TVD (Total Variation Diminishing) schemes derived from the Harten formalism for the numerical solution in 1D of various linear and non linear hyperbolic problems are now very popular -see also [19, 20, 23, 27, 30,31,33,35] and the references therein.

We refer to [32] and [28] for a presentation of major issues about the development of non dissipative schemes for linear advection in 2D for discontinuous flows - see also $[1,3,4,8,9,12,19,27,36]$. We particularly agree with Roe and Sidilkover [32]: "Genuinely multidimensional algorithms are only just beginning to be understood"; see also LeVeque ([27], p. 207). To our opinion this is strongly related to the lack of a general multidimensional VD (Variation Diminishing) estimate in 2D on arbitrary grids. Deriving general VD estimates for genuinely

\footnotetext{
Keywords and phrases. LVD estimate, Harten Formalism, linear advection, finite volume methods.

* This work was prepared when the second author was at the CEA.

1 Commissariat à l'Énergie Atomique, BP 12, 91680 Bruyères-le-Châtel, France. e-mail: Bruno.Despres@cea.fr

2 Laboratoire d'analyse numérique, Université de Paris VI, 175 rue du Chevaleret, 75013 Paris, France.

e-mail: despres@ann.jussieu.fr
} 
multidimensional schemes for various linear and non linear problems on arbitrary grids is challenging. This has been reported for instance by Lax (in "Systems of conservation laws and related topics" a conference celebrating Burt Wendroff's birthday): "Much efforts were spent on trying to devise TVD schemes for multidimensional conservation laws. The search is, of course, doomed to failure, since TV does not D in more than one dimension".

The current theory of approximation $[2,7,10,17]$ also suffers from the lack of a general BV (Bounded Variation) estimate, at least on general grids. It has motivated convergence study on arbitrary grids by means of measure value solutions $[15,34]$ at the numerical level, by the so called kinetic formulation [2,29], and via Kuznetsov error approximation [7, 17]. Nevertheless BV estimates exist for Cartesian meshes [24, 25], thus allowing to obtain optimal bounds for the numerical error on Cartesian meshes.

This work is an attempt to set a convenient framework for the development and analysis of genuinely multidimensional schemes on arbitrary grids. Our main result is an extension of TVD schemes and TVD estimate, called in the following LVD (Longitudinal Variation Diminishing) schemes and LVD estimate. Note that it does not enter in contradiction with Lax's remark: T is replaced by $\mathrm{L}, \mathrm{V}$ is unchanged, the gain is D. An abstract of the paper is the following.

"Let $\Omega \subset \mathbb{R}^{2}$ be a bounded domain, and let $\left(\Omega_{j}\right)_{j \in J}$ be an arbitrary mesh of $\Omega$. For any time evolution equation, consider a finite volume approximation: $\alpha_{j}$ (resp. $\left.\bar{\alpha}_{j}\right)$ is the current (resp. updated) numerical solution in the cell $\Omega_{j}$. Assume that a scheme allows to compute $\bar{\alpha}_{j}$ 's from the $\alpha_{j}$ 's, such that

$$
\forall j, \exists \gamma_{j} \in[0,1], \quad \bar{\alpha}_{j}=\left(1-\gamma_{j}\right) \alpha_{j}+\gamma_{j} \sum_{k \in J} p_{j k} \alpha_{k},
$$

where $P=\left(p_{j k}\right)$ is a stochastic matrix (cf. [37]):

$$
\sum_{k \in J} p_{j k}=1, \forall j \text { and } p_{j k} \geq 0 \forall j, k
$$

The dimension of the matrix $P$ is the number of cells, assumed to be finite and equal to card $(J)$."

Definition 1. We say that a scheme which may be written as (1) satisfies a generalized Harten formalism.

LVD estimate for generalized Harten formalism. For a scheme (1) there exists non-negative weights $\Lambda_{j} \geq 0$ (with at least one which is non zero) which depend on $P$ and do not depend on the $\gamma_{j}$ 's, $\alpha_{j}$ 's and $\bar{\alpha}_{j}$ 's, such that the estimate (2) holds

$$
\sum_{j \in J} \Lambda_{j}\left|\bar{\alpha}_{j}-\sum_{k \in J} p_{j k} \bar{\alpha}_{k}\right| \leq \sum_{j \in J} \Lambda_{j}\left|\alpha_{j}-\sum_{k \in J} p_{j k} \alpha_{k}\right|
$$

The weights $\Lambda=\left(\Lambda_{j}\right)$ are solution of a global eigenvector problem for the eigenvalue 1

$$
P^{t} \Lambda=\Lambda
$$

In the following, $P$ is such that (2) is an estimate of the Total Variation Longitudinally to the streamlines. This is the reason why we propose to retain this LVD (Longitudinal Variation Diminishing) terminology.

LVD estimate for linear advection. Consider 2D linear advection with periodic boundary conditions

$$
\partial_{t} \alpha+\vec{a} . \vec{\nabla} \alpha=0, \quad(t, x) \in[0, T] \times \Omega_{\mathrm{per}} .
$$

$\vec{a}$ is given and constant. Consider the standard finite volume upwind approximation on arbitrary grid of this problem: $\alpha_{j}$ is the current numerical solution in the cell $\Omega_{j} ; \bar{\alpha}_{j}$ is the updated numerical solution in the same cell. Denote $I^{-}(j)$ the set of neighboring incoming cells (i.e. $k \in I^{-}(j)$ if and only if $\left.m_{j k}=-\int_{\overline{\Omega_{j}}} \cap \overline{\Omega_{k}}\left(\vec{a}, \vec{n}_{j}\right)>0\right)$, and define $p_{j k}=\frac{m_{j k}}{\sum_{l \in I^{-}(j)} m_{j l}}$ if $k \in I^{-}(j)$ and $p_{j k}=0$ if $k \notin I^{-}(j)$. 
Then a) the upwind scheme may be rewritten as (1) using the matrix $P$ given above, b) for this particular matrix $P$ the weights $\Lambda_{j}(2)$ are given explicitly

$$
\Lambda_{j}=\sum_{l \in I^{-}(j)} m_{j l}
$$

As a consequence the LVD estimate (2) for the upwind scheme applied to linear advection may be rewritten as

$$
\sum_{j \in J}\left|\left(\sum_{k \in I^{-}(j)} m_{j k}\right) \bar{\alpha}_{j}-\sum_{k \in I^{-}(j)} m_{j k} \bar{\alpha}_{k}\right| \leq \sum_{j \in J}\left|\left(\sum_{k \in I^{-}(j)} m_{j k}\right) \alpha_{j}-\sum_{k \in I^{-}(j)} m_{j k} \alpha_{k}\right|
$$

In $1 \mathrm{D}$ one easily checks on simple examples $(a>0)$ that $p_{j k}=\delta_{j-1, k}$ and $\Lambda_{j}=1$. So $(2)$ is in $1 \mathrm{D}$

$$
\sum_{j \in J}\left|\bar{\alpha}_{j}-\bar{\alpha}_{j-1}\right| \leq \sum_{j \in J}\left|\alpha_{j}-\alpha_{j-1}\right|
$$

It explains why inequality (2) is a multidimensional generalization of the well known 1D TVD inequality (4). The upwind scheme discussed in the following is the simplest example of a scheme for which the generalized Harten formalism (1) is true.

These results need some comments. In dimension one, it is well known [21] that both definitions of the total variation, that is the continuous one and the discrete one (4), are equal when applied to a discrete profile. In dimension greater than one, both sides of (3) may be considered as very close to some numerical approximations of the $L^{1}$ norm of $\operatorname{div}(\alpha \vec{a})=\vec{a} \cdot \vec{\nabla} \alpha$, but these quantities are definitely different. So there is an important difference between dimension one and dimension greater than one. It explains why this paper is entirely written at the discrete level.

In Section 2, we introduce some notations: linear advection is, in this work, the model problem. In Section 3 we propose a 2D generalization on arbitrary grids of TVD schemes, based on an extension of the formalism [13, 26]. These schemes are non linear in the general case and satisfy a generalized Harten formalism. In Section 4 we derive a natural Variation Diminishing estimate (2) for this family of schemes on arbitrary grids. We propose to call it LVD estimate due to the presence of various weights. The proof of the LVD estimate relies on essentially three points: a) rewriting the generalized Harten formalism with a stochastic matrix $P$; b) the Perron-Frobenius Theorem for the study in the general case of the maximal left eigenvector of this matrix; c) explicit calculation of the maximal left eigenvector for the matrix $P$ defined by the numerical approximation of linear advection. Simple examples on square grids show that the LVD estimate is a natural extension on arbitrary grids of the TVD estimate. Finally in Section 5 and for the sake of completeness, a simple consequence of the LVD estimate on arbitrary grids is WBV (Weak Bounded Variation) estimates [17] with better constants (see also [10]). It gives a proof of weak convergence on $2 \mathrm{D}$ arbitrary uniformly regular triangular mesh for all linear non linear $L V D$ schemes defined in Section 3.

It is worthwhile to notice that the standard proof of convergence via WBV estimates assumes enough dissipation of the scheme -see [17] for a complete discussion. Our proof does not assume such a dissipation process: it is an important advantage of LVD estimates. It leaves place for the study of convergent non linear and non dissipative schemes for linear advection and transport equation on arbitrary grids (recent progress has been made on finite difference grids $[13,26])$. We delay to a forthcoming work the question of finding optimal non dissipative LVD schemes for "real computations". 


\section{Notations AND MODEL PROBLEM}

We consider the following linear advection model problem

$$
\begin{cases}\partial_{t} \alpha+\vec{a} \cdot \vec{\nabla} \alpha=0, & (t, x) \in[0, T] \times \Omega, \\ \alpha(t=0, x)=\alpha_{0}(x), & x \in \Omega\end{cases}
$$

For the sake of simplicity we consider the $2 \mathrm{D}$ case

$$
\Omega=[0,1] \times[0,1] \subset \mathbb{R}^{2},
$$

assume that $\vec{a} \neq 0$ is constant in space and time, and supplement (5) with periodic boundary conditions

$$
\begin{cases}\alpha\left(t, 0, x_{2}\right)=\alpha\left(t, 1, x_{2}\right), & \left(t, x_{2}\right) \in[0, T] \times[0,1], \\ \alpha\left(t, x_{1}, 0\right)=\alpha\left(t, x_{1}, 1\right), & \left(t, x_{1}\right) \in[0, T] \times[0,1] .\end{cases}
$$

Let $\left(\Omega_{j}\right)_{j \in J}$ be a finite mesh of $\Omega$

$$
\left\{\begin{array}{l}
\Omega_{j} \cap \Omega_{k}=\emptyset, \\
\cup_{j \in J} \overline{\Omega_{j}}=\bar{\Omega}=\Omega .
\end{array} \forall j, k, j \neq k\right.
$$

The shape of any cell is arbitrary polygonal. Most usual cases are square cells (finite difference) or triangle cells (finite volume).

Two cells are neighboring cells if and only if they have an edge in common (taking in account periodic boundary conditions). Each cell has a finite number of neighbors: $I(j)$ is the set of indices of the neighbors of cell $j$. The outgoing normal from $\Omega_{j}$ on the edge $\overline{\Omega_{j}} \cap \overline{\Omega_{k}}$ is denoted as $\vec{n}_{j k}$. Of course the outgoing normal from $\Omega_{j}$ is the opposite of the outgoing normal from $\Omega_{k}$ for $k \in I(j)$

$$
\vec{n}_{j k}+\vec{n}_{k j}=0
$$

Since the mesh is assumed to be made of triangles or squares, then the edges are straight lines and $\vec{n}_{j k}=-\vec{n}_{k j}$ is constant along $\overline{\Omega_{j}} \cap \overline{\Omega_{k}}$. We introduce some very natural notations

$$
\begin{cases}l_{j k}=l_{k j}=\mathbb{R} \text {-Lebesgue measure of } \overline{\Omega_{j}} \cap \overline{\Omega_{k}}, & \text { a length, } \\ s_{j}=\mathbb{R}^{2} \text {-Lebesgue measure of } \Omega_{j}, & \text { a surface. }\end{cases}
$$

We also define

$$
\left\{\begin{array}{l}
I^{+}(j)=\left\{k \in I(j) ;\left(\vec{a}, \vec{n}_{j k}\right)>0\right\} \\
I^{0}(j)=\left\{k \in I(j) ;\left(\vec{a}, \vec{n}_{j k}\right)=0\right\} \\
I^{-}(j)=\left\{k \in I(j) ;\left(\vec{a}, \vec{n}_{j k}\right)<0\right\}
\end{array}\right.
$$

and

$$
m_{j k}=m_{k j}=l_{j k}\left|\left(\vec{a}, \vec{n}_{j k}\right)\right|
$$

Here and in the rest of the paper $(.,$.$) denotes the standard scalar product. I^{+}(j)$ (resp. $I^{-}(j)$ ) is the set of outgoing (resp. incoming) cells from $\Omega_{j}$. An example on triangle is given in Figure 1. With all these notations a standard finite volume like method may be defined as

$$
s_{j} \frac{\bar{\alpha}_{j}-\alpha_{j}}{\Delta t}+\sum_{k \in I^{+}(j)} m_{j k} \alpha_{j k}-\sum_{k \in I^{-}(j)} m_{j k} \alpha_{j k}=0, \quad \forall j \in J
$$




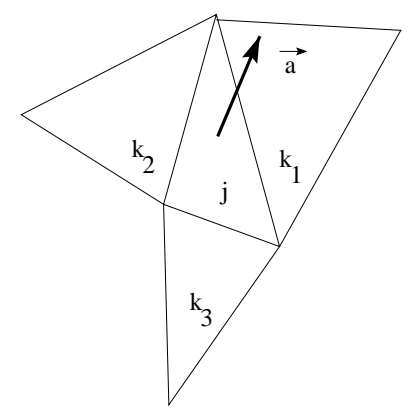

Figure $1 . I^{+}(j)=\left\{k_{2}, k_{3}\right\}, I^{-}(j)=\left\{k_{1}\right\}$.

where $m_{j k} \alpha_{j k}$ is the flux value integrated along the edge $\overline{\Omega_{j}} \cap \overline{\Omega_{k}}$, to be determined. In order to save notations the index of iteration $n$ has been omitted: $\alpha_{j}$ stands for the current value in the cell $\Omega_{j}, \alpha_{j}=\alpha_{j}^{n} ; \bar{\alpha}_{j}$ stands for the updated value in the same cell, $\bar{\alpha}_{j}=\alpha_{j}^{n+1}$; since we study explicit schemes, $\alpha_{j k}$ stands for $\alpha_{j k}^{n}$. In the following we consider symmetric values of the fluxes

$$
\alpha_{j k}=\alpha_{k j} \text { for } k \in I(j)
$$

thus the scheme (13) is conservative.

Of course the standard upwind value of the flux

$$
\alpha_{j k}=\alpha_{k} \text { for } k \in I^{-}(j)
$$

gives the well known upwind scheme

$$
s_{j} \frac{\bar{\alpha}_{j}-\alpha_{j}}{\Delta t}+\sum_{k \in I^{+}(j)} m_{j k} \alpha_{j}-\sum_{k \in I^{-}(j)} m_{j k} \alpha_{k}=0, \quad \forall j \in J .
$$

The following formula will play an important role in the analysis.

Lemma 1. One has the equality

$$
\sum_{k \in I^{+}(j)} m_{j k}=\sum_{k \in I^{-}(j)} m_{j k}, \forall j .
$$

It is a well-known consequence of the divergence theorem

$$
0=\int_{\Omega_{j}} \operatorname{div} \vec{a}=\int_{\partial \Omega_{j}}\left(\vec{a}, \vec{n}_{j k}\right)=\sum_{k \in I^{+}(j)} m_{j k}-\sum_{k \in I^{-}(j)} m_{j k} .
$$

It is straightforward to check that the upwind scheme (16) may be rewritten as

$$
\bar{\alpha}_{j}=\left(1-\gamma_{j}\right) \alpha_{j}+\gamma_{j} \sum_{k \in J} p_{j k} \alpha_{k}
$$

with

$$
\gamma_{j}=\frac{\Delta t \sum_{k \in I^{-}(j)} m_{j k}}{s_{j}}
$$


and

$$
\begin{cases}p_{j k}=\frac{m_{j k}}{\sum_{k \in I^{-}(j)} m_{j k}}, & \forall k \in I^{-}(j), \\ p_{j k}=0, & \forall k \notin I^{-}(j) .\end{cases}
$$

Assuming a CFL condition, then $0 \leq \gamma_{j} \leq 1$ for all $j$. It is clear from intuitive geometrical reasons that $\sum_{k \in I^{-}(j)} m_{j k}>0$, so the definition of $p_{j k}$ makes sense. See (65) in Appendix A for a rigorous proof.

\section{SOME NON LINEAR SCHEMES}

In this section we propose values of the fluxes other than the upwind ones (15). Our purpose is to show that the LVD estimate, consequence (next section) of the generalized Harten formalism (18), is not restricted to the upwind scheme. These new fluxes define non linear schemes, even if linear advection is a linear equation: it is already the case in the 1D TVD theory [19].

The construction is an extension on arbitrary grids of the recent work $[13,26]$ about non dissipative TVD schemes on regular grids. We assume that the fluxes $\alpha_{j k}$ have to satisfy a compatibility principle for all $k \in I^{+}(j)$, a compatibility principle for all $k \in I^{-}(j)$, plus a kind of $L^{\infty}$ estimate. Note however that the following compatibility principles play a minor role in this paper: they are just a simple way for developing non-linear explicit schemes which verify the generalized Harten formalism; consequently these compatibility principles are a simple way to derive explicit schemes which verify the LVD estimate.

\subsection{Compatibility principle for all $k \in I^{+}(j)$}

We impose to the flux $\alpha_{j k}$ to be a convex combination of $\alpha_{j}$ and $\alpha_{k}$. The compatibility principle means that the combination coefficient is the same for all $k \in I^{+}(j)$

$$
\alpha_{j k}=\left(1-\beta_{j}\right) \alpha_{j}+\beta_{j} \alpha_{k}, \quad \beta_{j} \in[0,1], \quad \forall k \in I^{+}(j), \quad \forall j \in J .
$$

This kind of flux is very similar to the well known MUSCL numerical flux $[16,36]$ and therein. However the formula proposed in this paper for the evaluation of $\beta_{j k}$ is different from those given in listed references: see (34). The advantage of our approach is the LVD estimate satisfied by the family of scheme. So even if the flux proposed below has been constructed mainly for completely different purposes than in [16] and [36], it may be considered as a member of the MUSCL family.

\subsection{Compatibility principle for all $k \in I^{-}(j)$}

We assume that the fluxes satisfy

$$
\left(\sum_{k \in I^{-}(j)} m_{j k}\right) \min \left(\alpha_{j}, \sum_{k \in J} p_{j k} \alpha_{k}\right) \leq \sum_{k \in I^{-}(j)} m_{j k} \alpha_{j k} \leq\left(\sum_{k \in I^{-}(j)} m_{j k}\right) \max \left(\alpha_{j}, \sum_{k \in J} p_{j k} \alpha_{k}\right),
$$

where

$$
\begin{cases}p_{j k}=\frac{m_{j k}}{\sum_{k \in I^{-}(j)} m_{j k}}, & \forall k \in I^{-}(j), \\ p_{j k}=0, & \forall k \notin I^{-}(j) .\end{cases}
$$

We will see in the following what this assumption means.

Since $p_{j k}=0 \forall k \notin I^{-}(j)$, the sum in (20) is restricted to $k \in I^{-}(j)$. Note the interesting property

$$
\sum_{k \in J} p_{j k}=1 \forall j, \quad p_{j k} \geq 0 \forall j, k .
$$


If ever only one index is in $I^{-}(j)$ (in other words $I^{-}(j)=\left\{k_{0}\right\}$ ), then $p_{j k_{0}}=\frac{m_{j k_{0}}}{m_{j k_{0}}}=1$. In this case (20) may be rewritten as

$$
\min \left(\alpha_{j}, \alpha_{k_{0}}\right) \leq \alpha_{j k} \leq \max \left(\alpha_{j}, \alpha_{k_{0}}\right)
$$

and is a direct consequence of (19). Thus the constraint (20) is active only if card $\left(I^{-}(j)\right)>1$.

A simple manner to enforce (20) is to adopt the convention:

$$
\text { if }\left(\sum_{k \in I^{-}(j)} m_{j k}\left(\alpha_{k}-\alpha_{j}\right)\right)\left(\alpha_{k^{\prime}}-\alpha_{j}\right)<0 \text { for some } k^{\prime} \in I^{-}(j),
$$

then we impose

$$
\beta_{k}=0 \forall k \in I^{-}(j)
$$

A interpretation of (20) is the following: if the upwind scheme (16) predicts an increasing (resp. decreasing) value of $\bar{\alpha}_{j}$ then all the incoming fluxes have to follow this prediction.

\section{3. $L^{\infty}$ estimate}

We would like to impose the following $L^{\infty}$ estimate

$$
\min \left(\alpha_{j}, \sum_{k \in J} p_{j k} \alpha_{k}\right) \leq \bar{\alpha}_{j} \leq \max \left(\alpha_{j}, \sum_{k \in J} p_{j k} \alpha_{k}\right) .
$$

Inequality (24) is indeed a $L^{\infty}$ stability estimate, since it implies

$$
\min \left(\alpha_{j}, \min _{k \in I^{-}(j)} \alpha_{k}\right) \leq \bar{\alpha}_{j} \leq \max \left(\alpha_{j}, \max _{k \in I^{-}(j)} \alpha_{k}\right) .
$$

Note that (25) is equivalent to the generalized Harten formalism. A simple way to enforce (24) for a general scheme (13) is to impose some constraints on the fluxes. We do this with the help of the formalism developed in $[14,26]$. First of all we use (13) and rewrite (24) as

$$
\sum_{k \in I^{-}(j)} m_{j k} \alpha_{j k}+\frac{s_{j}}{\Delta t}\left(\alpha_{j}-\max \left(\alpha_{j}, \sum_{k \in J} p_{j k} \alpha_{k}\right)\right) \leq \sum_{k \in I^{+}(j)} m_{j k} \alpha_{j k}
$$

and

$$
\sum_{k \in I^{+}(j)} m_{j k} \alpha_{j k} \leq \sum_{k \in I^{-}(j)} m_{j k} \alpha_{j k}+\frac{s_{j}}{\Delta t}\left(\alpha_{j}-\min \left(\alpha_{j}, \sum_{k \in J} p_{j k} \alpha_{k}\right)\right) .
$$

The compatibility principle (20) allows to eliminate incoming fluxes in (26-27). We derive a sufficient double inequality

$$
\left(\sum_{k \in I^{-}(j)} m_{j k}\right) \max \left(\alpha_{j}, \sum_{k \in J} p_{j k} \alpha_{k}\right)+\frac{s_{j}}{\Delta t}\left(\alpha_{j}-\max \left(\alpha_{j}, \sum_{k \in J} p_{j k} \alpha_{k}\right)\right) \leq \sum_{k \in I^{+}(j)} m_{j k} \alpha_{j k},
$$


and

$$
\sum_{k \in I^{+}(j)} m_{j k} \alpha_{j k} \leq\left(\sum_{k \in I^{-}(j)} m_{j k}\right) \min \left(\alpha_{j}, \sum_{k \in J} p_{j k} \alpha_{k}\right)+\frac{s_{j}}{\Delta t}\left(\alpha_{j}-\min \left(\alpha_{j}, \sum_{k \in J} p_{j k} \alpha_{k}\right)\right) .
$$

We thus have

Lemma 2. If (20) and (28-29) are true then (26-27) is true so the $L^{\infty}$ estimate (25) is true.

Note that (28-29) is an inequality only for the $\beta_{j}$ variable, due the compatibility principle (19) for $k \in I^{+}(j)$

$$
\sum_{k \in I^{+}(j)} m_{j k} \alpha_{j k}=\sum_{k \in I^{+}(j)} m_{j k} \alpha_{j}+\beta_{j} \sum_{k \in I^{+}(j)} m_{j k}\left(\alpha_{k}-\alpha_{j}\right)
$$

We rewrite (28-29) as

$$
\left(\frac{s_{j}}{\Delta t}-\sum_{k \in I^{-}(j)} m_{j k}\right)\left(\alpha_{j}-\max \left(\alpha_{j}, \sum_{k \in J} p_{j k} \alpha_{k}\right)\right) \leq \beta_{j} \sum_{k \in I^{+}(j)} m_{j k}\left(\alpha_{k}-\alpha_{j}\right)
$$

and

$$
\beta_{j} \sum_{k \in I^{+}(j)} m_{j k}\left(\alpha_{k}-\alpha_{j}\right) \leq\left(\frac{s_{j}}{\Delta t}-\sum_{k \in I^{-}(j)} m_{j k}\right)\left(\alpha_{j}-\min \left(\alpha_{j}, \sum_{k \in J} p_{j k} \alpha_{k}\right)\right)
$$

(recall Lem. 1: $\sum_{k \in I^{+}(j)} m_{j k}=\sum_{k \in I^{-}(j)} m_{j k}, \forall j$ ).

The following result states that at least $\beta_{j}=0$ (i.e. the upwind scheme) is a solution of (19), (20) and $(30-31)$. In other terms all inequalities are compatible.

Lemma 3. Assume that the CFL condition

$$
\frac{\sum_{k \in I^{+}(j)} m_{j k}}{s_{j}} \Delta t \leq 1, \quad \forall j \in J
$$

is satisfied. Then $\beta_{j}=0$ solves the inequalities (19), (20) and (30-31) for all $j \in J$.

Due to the CFL condition the left-hand side of (30) is non positive and the right-hand side of (31) is nonnegative. So $\beta_{j}=0$ is a solution.

However the upwind scheme $\left(\beta_{j}=0 \forall j\right)$ is not the only solution of inequalities (19), (20) and (30-31).

For the sake of simplicity consider a cartesian mesh (Fig. 2) and assume that a given numerical profile $\left(\alpha_{j}\right)_{j}$ is monotone, in the sense that

$$
\alpha_{k}<\alpha_{j}, \forall j, \forall k \in I^{-}(j) .
$$

One is frequently faced with this monotone situation in applications (or with the reverse situation $\alpha_{k}>\alpha_{j}$ ). In this case (22) is never true -in other words, (20) is automatically true. So it is sufficient to consider only (30) and (31). Now the term $\beta_{j} \sum_{k \in I^{+}(j)} m_{j k}\left(\alpha_{k}-\alpha_{j}\right)$ is the product of $\beta_{j}$ with a positive coefficient $\sum_{k \in I^{+}(j)} m_{j k}\left(\alpha_{k}-\alpha_{j}\right)$. Thanks to a strict CFL condition

$$
\frac{\sum_{k \in I^{+}(j)} m_{j k}}{s_{j}} \Delta t<1
$$




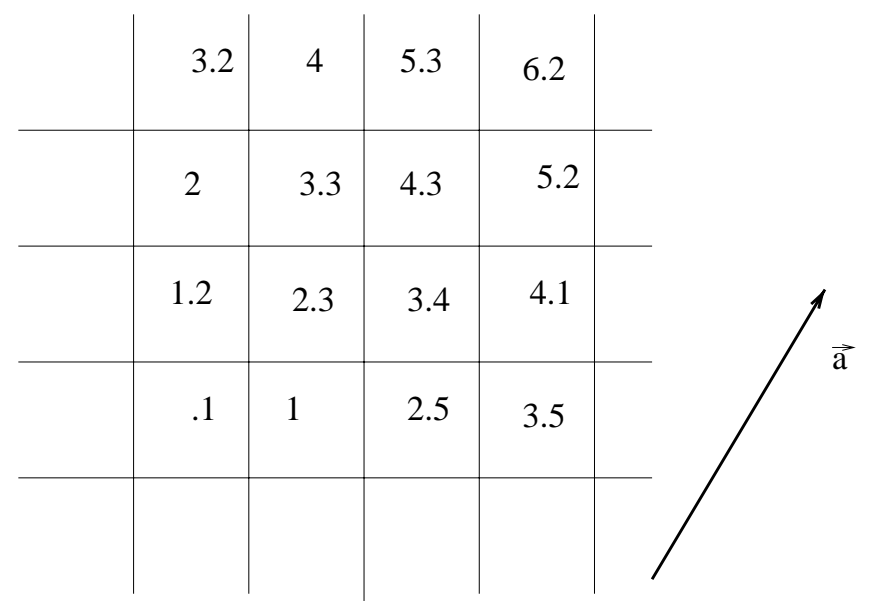

Figure 2. Example of monotone profile on a cartesian mesh.

and to (33), the left-hand side of (30) is negative and the right-hand side of (31) is positive. We deduce that all $\beta_{j}$ in the interval

$$
\left\{\begin{array}{l}
\beta_{j} \in\left[0, \beta_{j}^{\max }\right], \\
\beta_{j}^{\max }=\left(\frac{s_{j}}{\Delta t}-\sum_{k \in I^{-}(j)} m_{j k}\right) \frac{\alpha_{j}-\min \left(\alpha_{j}, \sum_{k \in J} p_{j k} \alpha_{k}\right)}{\sum_{k \in I^{+}(j)} m_{j k}\left(\alpha_{k}-\alpha_{j}\right)}>0
\end{array}\right.
$$

are other values such that (19), (20) and (30-31) are true. As a consequence the $L^{\infty}$ estimate (24) is true for such a choice of $\beta_{j}$. We have proved

Theorem 1. There exists other fluxes and other schemes than the upwind scheme (in the monotone situation (33), formula (34) is an example) such that (19), (20) and (30-31) are true for all $j \in J$. For these schemes it proves (24) which may be rewritten as (35).

\section{Longitudinal Variation Diminishing estimate}

Now we derive the LVD estimate for all schemes (13) such that the $L^{\infty}$ estimate (24) is true. Necessarily $\bar{\alpha}_{j}$ is a convex combination of the upper and lower bounds of (24).

$$
\forall j, \quad \exists \gamma_{j} \in[0,1], \quad \bar{\alpha}_{j}=\left(1-\gamma_{j}\right) \alpha_{j}+\gamma_{j} \sum_{k \in J} p_{j k} \alpha_{k}
$$

This is the generalized Harten formalism. In dimension 1, (35) implies the TVD estimate (4).

Let us gather all these quantities using some vector and matrix notations

$$
\begin{cases}P=\left(p_{j k}\right), & \text { a square matrix, } \\ I=\operatorname{diag}(1), & \text { the identity matrix, } \\ D=\operatorname{diag}\left(\gamma_{j}\right), & \text { a diagonal matrix, } \\ X=\left(\alpha_{j}\right), & \text { a vector, } \\ \bar{X}=\left(\bar{\alpha}_{j}\right), & \text { a vector, } \\ Y=\left(\left|((I-P) X)_{j}\right|\right), & \text { a vector, } \\ \bar{Y}=\left(\mid((I-P) \bar{X})_{j}\right), & \text { a vector. }\end{cases}
$$


All these objects are of dimension card $(J)$, number of cells. As already mentioned a matrix $P$ such that $p_{j k} \geq 0$, $\forall j, k$, and $\sum_{k \in J} p_{j k}=1, \forall j$ is called a stochastic matrix [37].

We rewrite (35) as

$$
\bar{X}=X-D(I-P) X
$$

Thus

$$
(I-P) \bar{X}=(I-P) X-(I-P) D(I-P) X=(I-D)(I-P) X+P D(I-P) X .
$$

Now we introduce the natural vector ordering

$$
\forall(X, Y) \in \mathbb{R}^{\operatorname{card}(J)}, \quad X \leq Y \text { if and only if } X_{j} \leq Y_{j} \forall j,
$$

and the natural matricial ordering

$$
\forall(P, Q) \in \mathbb{R}^{\operatorname{card}(J) \times \operatorname{card}(J)}, \quad P \leq Q \text { if and only if } P_{j k} \leq Q_{j k} \forall j, k .
$$

Taking the absolute value of each coefficient of the vector equation (36) we obtain

$$
\bar{Y} \leq(I-D) Y+P D Y .
$$

We have used the positivity of $P$ and the very important property $0 \leq D \leq I$, which is a consequence of its definition. In summary we have

$$
\bar{Y} \leq Y+(P-I) D Y .
$$

\subsection{Basic properties of the matrix $P$}

Since $P$ is a matrix with non-negative coefficients such that $\sum_{k \in I(j)} p_{j k}=1, \forall k$, then

$$
\|P\|_{\infty}=1
$$

for the induced matrix $l^{\infty}$ norm

$$
\|P X\|_{\infty}=\max _{X \neq 0} \frac{\|P X\|_{\infty}}{\|X\|_{\infty}}, \text { where }\|X\|_{\infty}=\max _{j}\left|X_{j}\right| .
$$

Since

$$
E=\left(\begin{array}{c}
1 \\
1 \\
\ldots \\
1
\end{array}\right)
$$

is clearly a right eigenvector of the matrix $P$ (indeed, $P E=E$ ), we know that there exists at least one left eigenvector of the matrix $P$, for the same eigenvalue. We denote this left eigenvector as $\Lambda$,

$$
\Lambda^{t} P=\Lambda^{t}
$$

If we assume that all the components of $\Lambda$ are non-negative,

$$
\Lambda_{j} \geq 0
$$


then we deduce from $(37)$

$$
(\Lambda, \bar{Y}) \leq(\Lambda, Y)+\left(\left(P^{t}-I\right) \Lambda, D Y\right)=(\Lambda, Y)
$$

where $(.,$.$) denotes the standard l^{2}$ scalar product. This is exactly what we call the Longitudinal Variation Diminishing estimate. Note that $\Lambda$ depends only on $P$ and not on $\left(\alpha_{j}\right)$. It depends only on $\vec{a}$ and on the mesh. In particular it is constant in time.

\subsection{More properties of the matrix $P$}

So the key point is to prove that the left eigenvector $\Lambda$ is indeed a non-negative vector. Reminiscence of the Krein-Rutman Theorem [11] or of the Perron-Frobenius Theorem [11,37] gives some hints that this property is true. Let us recall the following result which is a corollary of the Perron-Frobenius Theorem [37].

Theorem 2. Let $A \neq 0$ be a non-negative square matrix with non-negative coefficients

$$
A_{j k} \geq 0, \forall j, k \text {. }
$$

Then there exists one maximal real eigenvalue

$$
\lambda=\rho(A)>0,
$$

associated with a non-negative eigenvector $\Lambda \neq 0$

$$
\Lambda_{j} \geq 0 \forall j
$$

such that

$$
A \Lambda=\lambda \Lambda
$$

Applying Theorem 2 to $A=P^{t}$ and since $\rho\left(P^{t}\right)=\rho(P)=1$, it explains why $\Lambda_{j} \geq 0, \Lambda \neq 0$, is true $(\Lambda$ being defined by (40) and $\lambda=1$ ). If we assume moreover that the matrix $A$ is irreducible, the Perron-Frobenius Theorem states that $\Lambda>0$, i.e. $\Lambda_{j}>0$ for all $j$.

With straightforward notations we denote $X^{n}=\left(\alpha_{j}^{n}\right)$ the numerical solution of the scheme (13) at the $n$-th time step. We assume it enters the generalized Harten formalism:

$$
\forall n, \forall j, \quad \exists \gamma_{j}^{n} \in[0,1], \alpha_{j}^{n+1}=\left(1-\gamma_{j}^{n}\right) \alpha_{j}^{n}+\gamma_{j}^{n} \sum_{k \in J} p_{j k} \alpha_{k}^{n}
$$

$Y^{n}=\left|(I-P) X^{n}\right|$ is defined by

$$
Y_{j}^{n}=\left|\sum_{k \in J} p_{j k}\left(\alpha_{j}^{n}-\alpha_{k}^{n}\right)\right|
$$

Iterating in time (41) and since $\Lambda$ depends only on the constant matrix $P$, we get

Theorem 3. A numerical solution of any scheme verifying (42) satisfies the LVD (Longitudinal Variation Diminishing) estimate

$$
\left(\Lambda, Y^{n}\right) \leq\left(\Lambda, Y^{n-1}\right) \leq \ldots \leq\left(\Lambda, Y^{0}\right)
$$


that is

$$
\sum_{j \in J} \Lambda_{j}\left|\sum_{k \in J} p_{j k}\left(\alpha_{j}^{n}-\alpha_{k}^{n}\right)\right| \leq \sum_{j \in J} \Lambda_{j}\left|\sum_{k \in J} p_{j k}\left(\alpha_{j}^{0}-\alpha_{k}^{0}\right)\right|
$$

\subsection{More about the left eigenvector}

Thus the previously cited corollary of the Perron-Frobenius Theorem gives an abstract framework such that a generic diminishing estimate holds for every scheme which may be rewritten as (42). Hopefully it is possible, in the case of linear advection, to give the exact value of the weights.

Theorem 4. Consider the matrix P given by (21). Then a solution of (40) is

$$
\Lambda_{j}=\sum_{k \in I^{-}(j)} m_{j k}>0
$$

All $\Lambda_{j}$ 's defined by (45) are positive due to (64). The equation $P^{t} \Lambda=\Lambda$ means that

$$
\sum_{k \in J} p_{k j} \Lambda_{k}=\Lambda_{j}, \forall j
$$

that is

$$
\sum_{k \in I^{+}(j)}\left(\frac{m_{j k}}{\sum_{r \in I^{-}(k)} m_{k r}}\right) \Lambda_{k}=\Lambda_{j}, \quad \forall j
$$

We define

$$
\mu_{j}=\frac{\Lambda_{j}}{\sum_{r \in I^{-}(j)} m_{j r}}, \forall j
$$

Then we rewrite (46) as

$$
\sum_{k \in I^{+}(j)}\left(m_{k j}\right) \mu_{k}=\left(\sum_{r \in I^{-}(j)} m_{j r}\right) \mu_{j}, \forall j .
$$

Due to the divergence identity (17) one has

$$
\sum_{r \in I^{-}(j)} m_{j r}=\sum_{r \in I^{+}(j)} m_{j r}=\sum_{k \in I^{+}(j)} m_{k j} .
$$

Thus (47) is

$$
\sum_{k \in I^{+}(j)}\left(m_{k j}\right) \mu_{k}=\left(\sum_{k \in I^{+}(j)} m_{k j}\right) \mu_{j}, \forall j
$$

Finally $\mu_{j}=1$ for all $j$ is a solution. It proves (45). 


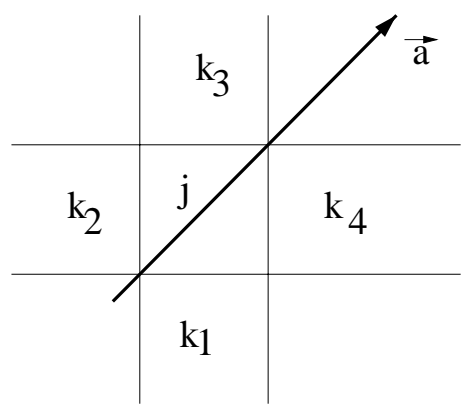

FiguRE 3. Example: $\vec{a}=(\sqrt{2} / 2, \sqrt{2} / 2)$.

Corollary 1. Consider a numerical solution of the scheme (13) such that (21) and (35) are true (assuming a CFL condition, the upwind scheme is an example of such a scheme). Then the following diminishing estimate holds

$$
\sum_{j \in J}\left|\left(\sum_{k \in I^{-}(j)} m_{j k}\right) \bar{\alpha}_{j}-\sum_{k \in I^{-}(j)} m_{j k} \bar{\alpha}_{k}\right| \leq \sum_{j \in J}\left|\left(\sum_{k \in I^{-}(j)} m_{j k}\right) \alpha_{j}-\sum_{k \in I^{-}(j)} m_{j k} \alpha_{k}\right| .
$$

The proof is by direct calculation: consider (44), use (45) and combine with the definition (21) of $P$.

\subsection{The matrix $P$ for square cells}

Here we discuss the simple example where the cells are squares. In some sense this example corresponds to finite differences. It is clear that in this case $\Lambda_{j}=\Lambda_{k}$ for all $j, k$ (see (45)). So the diminishing inequality simplifies into

$$
\sum_{j \in J}\left|\sum_{k \in J} p_{j k}\left(\alpha_{j}^{n}-\alpha_{k}^{n}\right)\right| \leq \sum_{j \in J}\left|\sum_{k \in J} p_{j k}\left(\alpha_{j}^{0}-\alpha_{k}^{0}\right)\right|
$$

We consider two cases.

a: Assume that $\vec{a}=(\sqrt{2} / 2, \sqrt{2} / 2)$. So

$$
p_{j k}=\frac{1}{2} \forall k \in I^{-}(j), \quad p_{j k}=0 \text { otherwise. }
$$

$p_{j k}$ is non zero if and only if the cell $k$ is immediately under or on the left of the cell $j$. In the example of Figure 3

$$
p_{j k_{1}}=p_{j k_{2}}=\frac{1}{2} \text { and } p_{j k_{3}}=p_{j k_{4}}=0 .
$$

By summation we thus obtain

$$
\sum_{j \in J}\left|\alpha_{j}^{n}-\frac{1}{2} \sum_{k \in I^{-}(j)} \alpha_{k}^{n}\right| \leq \sum_{j \in J}\left|\alpha_{j}^{0}-\frac{1}{2} \sum_{k \in I^{-}(j)} \alpha_{k}^{0}\right| .
$$

b: Assume that $\vec{a}=(1,0)$. So

$$
p_{j k}=1 k \in I^{-}(j), \quad p_{j k}=0 \text { otherwise. }
$$




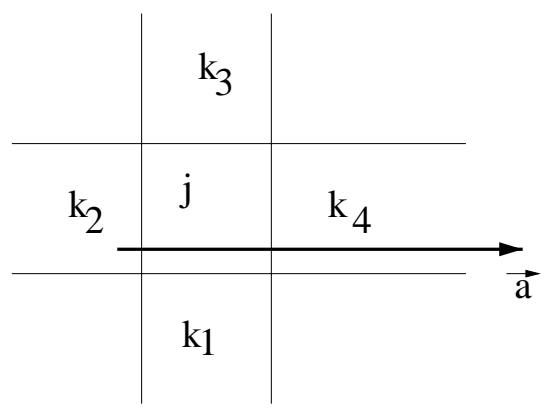

Figure 4. Example: $\vec{a}=(1,0)$.

$p_{j k}$ is non zero if and only if the cell $k$ is immediately on the left of the cell $j$. In the example of Figure 4

$$
p_{j k_{2}}=1 \text { and } p_{j k_{1}}=p_{j k_{3}}=p_{j k_{4}}=0 .
$$

We thus obtain

$$
\sum_{\text {lines }}\left(\sum_{j, k \text { neighboring on the line }}\left|\alpha_{j}^{n}-\alpha_{k}^{n}\right|\right) \leq \sum_{\text {lines }}\left(\sum_{j, k \text { neighboring on the line }}\left|\alpha_{j}^{0}-\alpha_{k}^{0}\right|\right) .
$$

In this case LVD is TVD only line by line.

Here we see that LVD is TVD longitudinally to the streamlines due to the weights $p_{j k}$ and $\left|\left(\vec{a}, \vec{n}_{j k}\right)\right|$. This is the reason why we propose to retain this LVD (Longitudinal Variation Diminishing) terminology.

\section{A PROOF OF WEAK CONVERGENCE FOR LVD SCHEMES}

A LVD scheme refers to a scheme (13) which satisfies the LVD estimates of Theorem 3, consequence for example of the generalized Harten formalism (42). Proving strong convergence with optimal rate of convergence for these kind of non linear schemes on arbitrary grids is still an open problem nowadays. Many researchers have stressed that non optimal bounds for the error are probably due to the lack of a BV estimate. Since the core of our work is precisely the derivation of such an LVD estimate for arbitrary grids, there is some hope that optimal bounds will take advantage of the approach developed in this work. Moreover optimal error estimates for monotone schemes need Kruzkov entropy inequalities (discussed for example in $[7,17,24,25]$ ), which are far from the scope of this paper.

From both examples on square grids (51-52) it is clear that LVD does not imply TVD: at most TVD line by line in the case (52). As a consequence it is not possible to rely on Helly Theorem (compact embedding of $B V \cap L^{1} \subset L^{1}$, see [19]) in order to prove strong convergence for general grids.

So we delay the question of strong convergence, and rely on an analysis of [17] in order to simply prove weak convergence via Weak Bounded Variations estimates.

An interest of the following proof is that we do not assume that the scheme is monotone as in [17]. The LVD estimate and $\alpha_{0} \in L^{\infty}(\Omega) \cap B V(\Omega)$ are sufficient.

More precise definition of uniformly regular meshes could be found in [5]. For the sake of simplicity, we consider in the following only meshes with triangle cells or quadrilateral cells.

Theorem 5. Be $\Omega=[0,1] \times[0,1] \subset \mathbb{R}^{2}$. Let us consider a sequence of triangular or quadrangular meshes with $\Delta x \rightarrow 0: \Delta x$ is a characteristic length of the mesh. We assume that the sequence of meshes is uniformly regular 
in the sense that

$$
\exists\left(c_{1}, c_{2}, c_{3}\right) \in\left(\mathbb{R}^{+, *}\right)^{3}
$$

such that for every mesh in the sequence

$$
l_{j k} \leq c_{1} \Delta x, c_{2} \Delta x^{2} \leq s_{j} \leq c_{3} \Delta x^{2}, \forall j, k
$$

Let $\alpha_{0} \in L^{\infty}(\Omega) \cap B V(\Omega)$. Let $\alpha_{\Delta x}$ be a sequence of numerical solutions, such that

a: the initial value is given by the total mass approximation

$$
\left(\alpha_{\Delta x}\right)_{j}^{0}=\frac{1}{s_{j}} \int_{\Omega_{j}} \alpha_{0}
$$

(note that $s_{j}$ and $\Omega_{j}$ depend on $\Delta x$ ),

b: for each $\Delta x,\left(\alpha_{\Delta x}\right)^{n}$ is given by the scheme (13-14),

c: the flux is a convex combination

$$
\min \left(\alpha_{j}, \alpha_{k}\right) \leq \alpha_{j k} \leq \max \left(\alpha_{j}, \alpha_{k}\right)
$$

as in (19),

d: the Generalized Harten Formalism (42) is true for all $j$ and $n$.

The upwind scheme (15-54) together with the CFL condition (32) is an example of such a sequence of numerical solutions. Another non linear example is given in Theorem 1.

Let us define

$$
\alpha_{\Delta x}=\sum_{j, n} \alpha_{j}^{n} \times 1_{j}^{n} \in L^{\infty}([0, T] \times \Omega),
$$

that is

$$
\left.\alpha_{\Delta x}(x, t)=\alpha_{j}^{n} \quad \forall(x, t) \in \Omega_{j} \times\right] n \Delta t,(n+1) \Delta t[
$$

Let $\alpha \in L^{\infty}([0, T] \times \Omega)$ be the solution of

$$
\left\{\begin{array}{l}
\partial_{t} \alpha+\vec{a} \cdot \nabla \alpha=0 \\
\alpha(0, x)=\alpha_{0}(x)
\end{array}\right.
$$

with periodic boundary conditions: $\alpha(x, t)=\alpha_{0}(t-(\vec{a}, x))$. Then $\alpha_{\Delta x}$ converges weakly in $L^{\infty, *}([0, T] \times \Omega)$ to $\alpha$, that is,

$$
\forall \varphi \in L^{1}([0, T] \times \Omega), \lim _{\Delta x \rightarrow 0}\left(\int_{[0, T] \times \Omega}\left(\alpha_{\Delta x}-\alpha\right) \varphi \mathrm{d} x \mathrm{~d} t\right)=0
$$

In [17] a similar result of convergence is based on the first of these two following WBV estimates

$$
\sum_{p=0}^{p=Q} \Delta t \sum_{j \in J} \sum_{k \in I^{-}(j)} m_{j k}\left|\alpha_{j}^{p}-\alpha_{k}^{p}\right| \leq \frac{C_{1}}{\Delta x^{\frac{1}{2}}}, Q \Delta t=T
$$


and

$$
\sum_{p=0}^{p=Q} \sum_{j \in J} s_{j}\left|\alpha_{j}^{p+1}-\alpha_{j}^{p}\right| \leq \frac{C_{1}}{\Delta x^{\frac{1}{2}}} .
$$

The constant $C_{1}>0$ depends only on $\alpha_{0}, \vec{a}, T=Q \Delta t$ and on $\left(c_{1}, c_{2}, c_{3}\right)$ characterizing the regularity of the mesh. In fact in [17] $C_{1}$ depends also on an additional parameter which states that the CFL number must be strictly less than one. Moreover it is possible for the upwind scheme (15) to replace the bound $\frac{C_{1}}{\Delta x^{\frac{1}{2}}}$ in $(58)$ by $C_{1}$. Thus the constant in our WBV estimates (57-58) is slightly better than in the original work [17]. Note that we impose $\alpha_{0} \in L^{\infty}(\Omega) \cap B V(\Omega)$ in our hypothesis while [17] needs only $\alpha_{0} \in L^{\infty}(\Omega)$ : it is a consequence of the use of the LVD estimate.

Lemma 4. Assume all the hypothesis of the previous theorem. Then the WBV estimates (57-58) are true with a bound $C_{1}>0$ which depends only on $\alpha_{0}, \vec{a}, T=Q \Delta t$ and $\left(c_{1}, c_{2}, c_{3}\right)$.

Under these hypotheses the LVD estimate (49) is true for all $n$ : it is a consequence of the generalized Harten formalism (42). Next to prove the WBV estimates we use the LVD estimate

$$
\begin{aligned}
& \sum_{j \in J}\left|\sum_{k \in I^{-}(j)} m_{j k}\left(\alpha_{j}^{n}-\alpha_{k}^{n}\right)\right| \leq \sum_{j \in J}\left|\sum_{k \in I^{-}(j)} m_{j k}\left(\alpha_{j}^{0}-\alpha_{k}^{0}\right)\right| \\
& \leq|\vec{a}|\left(\sum_{j \in J} \sum_{k \in I^{-}(j)} l_{j k}\left|\alpha_{j}^{0}-\alpha_{k}^{0}\right|\right)=|\vec{a}| \times\left\|\left(\alpha_{\Delta x}\right)^{0}\right\|_{B V(\Omega)} .
\end{aligned}
$$

We have used the definition of the BV semi-norm, true for a piecewise constant function (see [18])

$$
\left\|\alpha_{\Delta x}\right\|_{B V(\Omega)}=\sum_{j \in J} \sum_{k \in I^{-}(j) \cup I^{0}(j)} l_{j k}\left|\alpha_{j}-\alpha_{k}\right| .
$$

Here we use the continuity of the $L^{2}$ projection on arbitrary uniformly regular grids in BV space [17] (a selfconsistent proof is given in Appendix 5):

$$
\exists C_{2}>0, \quad\left\|\left(\alpha_{\Delta x}\right)^{0}\right\|_{B V(\Omega)} \leq C_{2}\left\|\alpha_{0}\right\|_{B V(\Omega)}, \quad \forall \Delta x>0
$$

Thus

$$
\sum_{j \in J}\left|\sum_{k \in I^{-}(j)} m_{j k}\left(\alpha_{j}^{n}-\alpha_{k}^{n}\right)\right| \leq C_{3} .
$$

Now let us define

$$
\alpha_{\Delta x}^{n}=\sum_{n} \alpha_{j}^{n} \times 1_{\Omega_{j}} \in L^{\infty}(\Omega)
$$

that is

$$
\alpha_{\Delta x}^{n}(x, t)=\alpha_{j}^{n} \quad \forall x \in \Omega_{j}
$$


Then we use $\left\|\alpha_{\Delta x}^{n}\right\|_{L^{\infty}} \leq\left\|\alpha_{0}\right\|_{L^{\infty}}$ (which is a consequence of (25) and (54)) and a discrete integration by part (see formula (67) in Appendix 5) to obtain

$\sum_{j \in J} \sum_{k \in I^{-}(j)} m_{j k}\left(\alpha_{j}^{n}-\alpha_{k}^{n}\right)^{2}=2 \sum_{j \in J} \sum_{k \in I^{-}(j)} m_{j k}\left(\alpha_{j}^{n}-\alpha_{k}^{n}\right) \alpha_{j}^{n} \leq 2\left\|\left(\alpha_{\Delta x}\right)^{n}\right\|_{L^{\infty}} \times \sum_{j \in J}\left|\sum_{k \in I^{-}(j)} m_{j k}\left(\alpha_{j}^{n}-\alpha_{k}^{n}\right)\right| \leq C_{4}$.

From the Cauchy-Schwarz inequality one has

$$
\sum_{j \in J} \sum_{k \in I^{-}(j)} m_{j k}\left|\alpha_{j}^{n}-\alpha_{k}^{n}\right| \leq\left(\sum_{j \in J} \sum_{k \in I^{-}(j)} m_{j k}\right)^{\frac{1}{2}}\left(\sum_{j \in J} \sum_{k \in I^{-}(j)} m_{j k}\left(\alpha_{j}^{n}-\alpha_{k}^{n}\right)^{2}\right)^{\frac{1}{2}} .
$$

Inequality (66) of the appendix, true for a triangular or quadrangular uniformly regular mesh, gives

$$
\sum_{j \in J} \sum_{k \in I^{-}(j)} m_{j k}\left|\alpha_{j}^{n}-\alpha_{k}^{n}\right| \leq \frac{C_{5}}{\Delta x^{\frac{1}{2}}}
$$

After summation in time we obtain (57).

Concerning (58): it is a direct consequence of (60), (61) and the definition of the scheme

$$
s_{j}\left(\alpha_{j}^{n+1}-\alpha_{j}^{n}\right)=-\Delta t \sum_{k \in I^{-}(j)} m_{j k}\left(\alpha_{j}^{n}-\alpha_{k}^{n}\right)-\Delta t \sum_{k \in I^{+}(j)} m_{j k}\left(\alpha_{j k}^{n}-\alpha_{j}^{n}\right)+\Delta t \sum_{k \in I^{-}(j)} m_{j k}\left(\alpha_{j k}^{n}-\alpha_{k}^{n}\right) .
$$

The first right-hand side contribution is bounded by (60). Since the flux is a convex combination of the upwind and downwind values (compatibility principle (19)), the second and third right hand side contributions are bounded thanks to (61). It gives

$$
\sum_{j \in J} s_{j}\left|\alpha_{j}^{n+1}-\alpha_{j}^{n}\right| \leq 3 \Delta t \sum_{j \in J} \sum_{k \in I^{-}(j)} m_{j k}\left|\alpha_{j}^{n}-\alpha_{k}^{n}\right| \leq \frac{C_{6}}{\Delta x^{\frac{1}{2}}} \Delta t
$$

After summation in time it gives (58). Considering the upwind scheme the second and third right hand side contributions vanish: it gives for the upwind scheme only

$$
\sum_{j \in J} s_{j}\left|\alpha_{j}^{n+1}-\alpha_{j}^{n}\right| \leq \Delta t \sum_{j \in J}\left|\sum_{k \in I^{-}(j)} m_{j k}\left(\alpha_{j}^{n}-\alpha_{k}^{n}\right)\right| \leq C_{7} \Delta t
$$

which gives a sharper estimate $\left(C_{7}\right.$ instead of $\left.\frac{C_{6}}{\Delta x^{\frac{1}{2}}}\right)$ in (58). Defining $C_{1}=\max \left(C_{5}, C_{6}, C_{7}\right)$ it ends the proof of Lemma 4.

\section{Final proof of Theorem 5.}

Since $\alpha_{0} \in L^{\infty}(\Omega)$ then the approximation $\alpha_{\Delta x}$ is uniformly bounded in $L^{\infty}([0, T] \times \Omega)$

$$
\exists C_{8}>0,\left\|\alpha_{\Delta x}\right\|_{L^{\infty}([0, T] \times \Omega)} \leq C_{8} .
$$

It implies the existence of $\alpha \in L^{\infty}([0, T] \times \Omega)$ such that $\alpha_{\Delta x}$ converges to $\alpha$ in the $L^{\infty, *}([0, T] \times \Omega)$ sense (up to an extracted subsequence). Now essentially copying the proof in [17] it gives the result $(55)$. Let $\varphi \in C^{1}([0, T] \times \Omega)$ be a smooth test function. We assume that $\varphi(x, T)=0 \forall x \in \Omega$. We multiply (13) by $\frac{\Delta t}{s_{j}} \varphi(x,(n+1) \Delta t)$, integrate over $x \in \Omega_{j}$ and sum for all $j$ and for all $0 \leq n \leq Q=\frac{T}{\Delta t}$. It gives

$$
A_{\Delta x}+B_{\Delta x}+D_{\Delta x}=0
$$


where

$$
\begin{aligned}
& A_{\Delta x}=\sum_{n} \sum_{j}\left(\alpha_{j}^{n+1}-\alpha_{j}^{n}\right) \int_{\Omega_{j}} \varphi(x,(n+1) \Delta t) \mathrm{d} x, \\
& B_{\Delta x}=\sum_{n} \sum_{j} \frac{\Delta t}{s_{j}}\left(\sum_{k \in I^{-}(j)} m_{j k}\left(\alpha_{j}^{n}-\alpha_{k}^{n}\right)\right) \int_{\Omega_{j}} \varphi(x,(n+1) \Delta t) \mathrm{d} x,
\end{aligned}
$$

and

$$
D_{\Delta x}=\sum_{n} \sum_{j}\left(\sum_{k \in I^{+}(j)} m_{j k}\left(\alpha_{j k}^{n}-\alpha_{j}^{n}\right)-\sum_{k \in I^{-}(j)} m_{j k}\left(\alpha_{j k}^{n}-\alpha_{k}^{n}\right)\right) \frac{\Delta t}{s_{j}} \int_{\Omega_{j}} \varphi(x,(n+1) \Delta t) \mathrm{d} x .
$$

Following [17] we note that

$$
\begin{aligned}
A_{\Delta x}= & -\int_{[0, T]} \int_{\Omega} \alpha_{\Delta x}(x, t) \partial_{t} \varphi(x, t) \mathrm{d} x \mathrm{~d} t-\int_{\Omega} \alpha_{0}(x) \varphi(x, 0) \mathrm{d} x \\
& +\sum_{j \in J} \int_{\Omega_{j}} \alpha_{0}(x)\left(\varphi(x, 0)-\frac{1}{s_{j}} \int_{\Omega_{j}} \varphi(y, 0) \mathrm{d} y\right) \mathrm{d} x .
\end{aligned}
$$

Since $\alpha_{\Delta x}$ converges to $\alpha$ in the $L^{\infty, *}([0, T] \times \Omega)$ sense, we get

$$
A_{\Delta x} \rightarrow-\int_{[0, T]} \int_{\Omega} \alpha(x, t) \partial_{t} \varphi(x, t) \mathrm{d} x \mathrm{~d} t-\int_{\Omega} \alpha_{0}(x) \varphi(x, 0) \mathrm{d} x \quad \text { as } \Delta x \rightarrow 0
$$

(recall that $\partial_{t} \varphi \in L^{1}$ ). Similarly we have

$$
\begin{aligned}
B_{\Delta x}= & -\sum_{n} \int_{n \Delta t}^{(n+1) \Delta t} \int_{\Omega} \alpha_{\Delta x}(x, t) \vec{a} . \vec{\nabla} \varphi(x, t) \mathrm{d} x \mathrm{~d} t+\sum_{n} \sum_{j} \Delta t\left(\sum_{k \in I^{-}(j)} m_{j k}\left(\alpha_{j}^{n}-\alpha_{k}^{n}\right)\right. \\
& \left.\times\left(\frac{1}{s_{j}} \int_{\Omega_{j}} \varphi(x,(n+1) \Delta t) \mathrm{d} x-\frac{1}{l_{j k} \Delta t} \int_{n \Delta t}^{(n+1) \Delta t} \int_{\overline{\Omega_{j}} \cap \overline{\Omega_{k}}} \varphi(x, t) \mathrm{d} l \mathrm{~d} t\right)\right) .
\end{aligned}
$$

Since $\varphi$ is smooth there exists $C_{9}>0$ such that

$$
\left|\frac{1}{s_{j}} \int_{\Omega_{j}} \varphi(x,(n+1) \Delta t) \mathrm{d} x-\frac{1}{l_{j k} \Delta t} \int_{n \Delta t}^{(n+1) \Delta t} \int_{\overline{\Omega_{j}} \cap \overline{\Omega_{k}}} \varphi(x, t) \mathrm{d} l \mathrm{~d} t\right| \leq C_{9} \Delta x, \quad \forall j, k .
$$

Thus using (60)

$$
\begin{gathered}
\left|\sum_{n} \sum_{j} \Delta t\left(\sum_{k \in I^{-}(j)} m_{j k}\left(\alpha_{j}^{n}-\alpha_{k}^{n}\right)\left(\frac{1}{s_{j}} \int_{\Omega_{j}} \varphi(x,(n+1) \Delta t) \mathrm{d} x-\frac{1}{l_{j k} \Delta t} \int_{n \Delta t}^{(n+1) \Delta t} \int_{\overline{\Omega_{j}} \cap \overline{\Omega_{k}}} \varphi(x, t) \mathrm{d} l \mathrm{~d} t\right)\right)\right| \\
\leq \frac{C_{1}}{\Delta x^{\frac{1}{2}}} C_{9} \Delta x \leq C_{10} \Delta x^{\frac{1}{2}}
\end{gathered}
$$


Since $\alpha_{\Delta x}$ converges to $\alpha$ in the $L^{\infty, *}([0, T] \times \Omega)$ sense, we get

$$
B_{\Delta x} \rightarrow-\int_{[0, T]} \int_{\Omega} \alpha(x, t) \vec{a} \cdot \vec{\nabla} \varphi(x, t) \mathrm{d} x \mathrm{~d} t \quad \text { as } \Delta x \rightarrow 0 .
$$

If we assume that $\lim _{\Delta x \rightarrow 0} D_{\Delta x}=0$, then

$$
\begin{gathered}
-\int_{[0, T]} \int_{\Omega} \alpha(x, t) \partial_{t} \varphi(x, t) \mathrm{d} x \mathrm{~d} t-\int_{\Omega} \alpha_{0}(x) \varphi(x, 0) \mathrm{d} x-\int_{[0, T]} \int_{\Omega} \alpha(x, t) \vec{a} . \vec{\nabla} \varphi(x, t) \mathrm{d} x \mathrm{~d} t=0, \\
\forall \varphi \in C^{1}([0, T] \times \Omega), \varphi(T)=0 .
\end{gathered}
$$

Thus it proves that $\alpha$ is the weak solution of (55).

So it remains to prove that the extra term $D_{\Delta x}$ tends to 0 . We sum (62) by part

$$
D_{\Delta x}=\sum_{n} \Delta t \sum_{k \in I^{+}(j)} m_{j k}\left(\alpha_{j k}^{n}-\alpha_{j}^{n}\right)\left(\frac{1}{s_{j}} \int_{\Omega_{j}} \varphi(x,(n+1) \Delta t) \mathrm{d} x-\frac{1}{s_{k}} \int_{\Omega_{k}} \varphi(x,(n+1) \Delta t) \mathrm{d} x\right) .
$$

Since $\varphi$ is smooth

$$
\exists C_{11}>0, \quad\left|\frac{1}{s_{j}} \int_{\Omega_{j}} \varphi(x,(n+1) \Delta t) \mathrm{d} x-\frac{1}{s_{k}} \int_{\Omega_{k}} \varphi(x,(n+1) \Delta t) \mathrm{d} x\right| \leq C_{11} \Delta x .
$$

Combining with $(60)$ we get

$$
\left|D_{\Delta x}\right| \leq \sum_{p=0}^{Q} \Delta t \frac{C_{1}}{\Delta x^{\frac{1}{2}}} C_{11} \Delta x \leq C_{12} \Delta x^{\frac{1}{2}} .
$$

It implies $\lim _{\Delta x \rightarrow 0} D_{\Delta x}=0$ and ends the proof of Theorem 5.

Acknowledgements. Both authors deeply thank the referee for many valuable remarks which have helped to improve the clarity of this work.

\section{ApPendix A. Regular GRIDS}

For the sake of completeness, we prove here some elementary properties about uniformly regular triangular or quadrangular grids. More precise definition of uniformly regular meshes may be found in [5].

Let us consider a sequence of meshes with $\Delta x \rightarrow 0: \Delta x$ is a characteristic length of the mesh. We assume that the sequence of meshes is uniformly regular in the sense that

$$
\exists\left(c_{1}, c_{2}, c_{3}\right) \in\left(\mathbb{R}^{+, *}\right)^{3} \text {, such that } l_{j k} \leq c_{1} \Delta x, c_{2} \Delta x^{2} \leq s_{j} \leq c_{3} \Delta x^{2}, \forall j, k .
$$

First we prove that such meshes satisfy

$$
0<D_{1} \sum_{l \in I^{-}(k)} m_{k l} \leq \sum_{l \in I^{-}(j)} m_{j l} \leq D_{2} \sum_{l \in I^{-}(k)} m_{k l}, \forall j, k,
$$

with $D_{1}$ and $D_{2}$ independent of the characteristic length of the mesh $\Delta x$.

Let $\vec{f}_{j}(\mathbf{x})=\left(\mathbf{x}-\mathbf{x}_{j}, \vec{a}\right) \vec{a} \in \mathbb{R}^{2}$ be a linear vectorial function of $\mathbf{x} \in \mathbb{R}^{2}$. Here $\mathbf{x}_{j} \in \mathbb{R}^{2}$ is the coordinate of any point inside the triangle $\Omega_{j}$. ¿From the divergence theorem we get

$$
\int_{\Omega_{j}} \operatorname{div} \vec{f}_{j}=\int_{\partial \Omega_{j}} \vec{f}_{j} \cdot \vec{n}_{j}
$$


where $\vec{n}_{j}$ is the outgoing normal. It is equivalent to

$$
s_{j}(\vec{a}, \vec{a})=\sum_{k \in I(j)}\left(\vec{a}, \vec{n}_{j k}\right) \int_{\partial \Omega_{j}}\left(\mathbf{x}-\mathbf{x}_{j}, \vec{a}\right) .
$$

Due to the various constants in (53) or (63) we obtain

$$
\begin{aligned}
c_{2} \Delta x^{2}(\vec{a}, \vec{a}) & \leq\left(\sum_{k \in I(j)}\left|\left(\vec{a}, \vec{n}_{j k}\right)\right| l_{j k}\right) \max _{\mathbf{x} \in \partial \Omega_{j}}\left|\left(\mathbf{x}-\mathbf{x}_{j}, \vec{a}\right)\right| \\
& \leq D_{3} \Delta x \sum_{k \in I(j)}\left|\left(\vec{a}, \vec{n}_{j k}\right)\right| l_{j k}
\end{aligned}
$$

(recall that the number of faces for each cell is less than 4).

Since

$$
\begin{aligned}
2 \sum_{k \in I^{-}(j)}\left|\left(\vec{a}, \vec{n}_{j k}\right)\right| l_{j k} & =\sum_{k \in I^{+}(j)}\left|\left(\vec{a}, \vec{n}_{j k}\right)\right| l_{j k}+\sum_{k \in I^{-}(j)}\left|\left(\vec{a}, \vec{n}_{j k}\right)\right| l_{j k} \\
& =\sum_{k \in I(j)}\left|\left(\vec{a}, \vec{n}_{j k}\right)\right| l_{j k}
\end{aligned}
$$

we deduce

$$
\exists c_{4}>0, \quad 0<c_{4} \Delta x \leq \sum_{k \in I^{-}(j)}\left|\left(\vec{a}, \vec{n}_{j k}\right)\right| l_{j k}, \forall j .
$$

On the other hand $\sum_{k \in I^{-}(j)}\left|\left(\vec{a}, \vec{n}_{j k}\right)\right| l_{j k}$ is bounded from above

$$
\exists c_{5}>0, \sum_{k \in I^{-}(j)}\left|\left(\vec{a}, \vec{n}_{j k}\right)\right| l_{j k} \leq c_{5} \Delta x, \quad \forall j .
$$

It proves (64) with uniform constants $D_{1}=\frac{c_{4}}{c_{5}}$ and $D_{2}=\frac{c_{5}}{c_{4}}$.

For a uniformly regular triangular or quadrangular mesh, we bound the number of cells

$$
\operatorname{card}(J) \leq \frac{\operatorname{meas}(\Omega)}{c_{2} \Delta x^{2}} \leq \frac{D_{4}}{\Delta x^{2}}
$$

It implies that

$$
\begin{aligned}
\left(\sum_{j \in J} \sum_{k \in I^{-}(j)} m_{j k}\right)^{\frac{1}{2}} & \leq\left(\frac{D_{4}}{\Delta x^{2}} \max _{j \in J} \sum_{k \in I^{-}(j)} m_{j k}\right)^{\frac{1}{2}} \\
& \leq\left(\frac{D_{4}}{\Delta x^{2}}|\vec{a}| D_{5} c_{1} \Delta x\right)^{\frac{1}{2}}
\end{aligned}
$$

where $D_{5}$ is 3 (resp. 4) for triangular (resp. quadrangular) meshes. Thus

$$
\left(\sum_{j \in J} \sum_{k \in I^{-}(j)} m_{j k}\right)^{\frac{1}{2}} \leq \frac{D_{6}}{\Delta x^{\frac{1}{2}}} .
$$

Here the constant $D_{6}$ depends on meas $(\Omega), \vec{a}$ and $C_{1}$.

\section{Appendix B. A Discrete integration By PART FORMUla}

Here we need to prove

$$
\sum_{j \in J} \sum_{k \in I^{-}(j)} m_{j k}\left(x_{j}-x_{k}\right)^{2}=2 \sum_{j \in J} \sum_{k \in I^{-}(j)} m_{j k}\left(x_{j}-x_{k}\right) x_{j}
$$


which is used in the proof of Lemma 4. One has

$$
\begin{aligned}
\sum_{j \in J} \sum_{k \in I^{-}(j)} m_{j k}\left(x_{j}-x_{k}\right) x_{j} & =\sum_{j \in J} \sum_{k \in I^{-}(j)} m_{j k} x_{j}^{2}-\sum_{j \in J} \sum_{k \in I^{-}(j)} m_{j k} x_{k} x_{j} \\
& =\sum_{j \in J} \sum_{k \in I^{-}(j)} m_{j k} x_{j}^{2}-\sum_{j \in J} \sum_{k \in I^{-}(j)} m_{j k}\left(\frac{1}{2} x_{j}^{2}-\frac{1}{2}\left(x_{j}-x_{k}\right)^{2}+\frac{1}{2} x_{k}^{2}\right) \\
& =\frac{1}{2} \sum_{j \in J} \sum_{k \in I^{-}(j)} m_{j k} x_{j}^{2}+\frac{1}{2} \sum_{j \in J} \sum_{k \in I^{-}(j)} m_{j k}\left(x_{j}-x_{k}\right)^{2}-\frac{1}{2} \sum_{j \in J} \sum_{k \in I^{-}(j)} m_{j k} x_{k}^{2} .
\end{aligned}
$$

Once more due to the divergence lemma

$$
\begin{aligned}
\frac{1}{2} \sum_{j \in J} \sum_{k \in I^{-}(j)} m_{j k} x_{k}^{2} & =\frac{1}{2} \sum_{k \in J} \sum_{j \in I^{-}(k)} m_{j k} x_{j}^{2} \\
& =\frac{1}{2} \sum_{j \in J} \sum_{k \in I^{+}(j)} m_{j k} x_{j}^{2}=\frac{1}{2} \sum_{j \in J} \sum_{k \in I^{-}(j)} m_{j k} x_{j}^{2} .
\end{aligned}
$$

Finally we obtain after simplification

$$
\sum_{j \in J} \sum_{k \in I^{-}(j)} m_{j k}\left(x_{j}-x_{k}\right) x_{j}=\frac{1}{2} \sum_{j \in J} \sum_{k \in I^{-}(j)} m_{j k}\left(x_{j}-x_{k}\right)^{2}
$$

which is (67).

\section{Appendix C. Continuity of the $L^{2}$ projection into BV space}

Here we have to prove (59).

This is a standard property, well known in finite element space, see [6] and references therein. However and since most of previous proof of convergence for finite volume approximations avoided the $B V$ framework [2,7], we give here a simple proof. The reader is invited to refer to [17] where a similar property is proved for implicit schemes.

We assume all the hypothesis of Appendix A and split the proof in three steps.

a: Let us consider a given mesh in a sequence of uniformly regular meshes. For a given $M=(x, y) \in \Omega$ and a given $R \in \mathbb{R}^{+}$, we denote as $\mathcal{N}(M, R)$ the number of cells $\Omega_{j}$ in the mesh such that $\Omega_{j} \subset \mathcal{B}(M, R)$ where $\mathcal{B}(M, R)$ stands for the ball centered at $M$ with radius $R$. We claim that

$$
\begin{gathered}
\forall E_{1}>0, \exists E_{2}\left(E_{1}\right)>0 \text { independent of the mesh size } \Delta x \\
\text { such that } \forall M \in \Omega \text {, we have } \mathcal{N}\left(M, E_{1} \Delta x\right) \leq E_{2}\left(E_{1}\right) .
\end{gathered}
$$

It is due to

$$
\begin{aligned}
\operatorname{meas}\left(\mathcal{B}\left(M, E_{1} \Delta x\right)\right) & =\pi\left(E_{1} \Delta x\right)^{2} \geq \sum_{\Omega_{j} \subset \mathcal{B}\left(M, E_{1} \Delta x\right)} \operatorname{meas}\left(\Omega_{j}\right) \\
& \geq \sum_{\Omega_{j} \subset \mathcal{B}\left(M, E_{1} \Delta x\right)} c_{2} \Delta x^{2}=\mathcal{N}\left(M, E_{1} \Delta x\right) c_{2} \Delta x^{2} .
\end{aligned}
$$

Thus

$$
\mathcal{N}\left(M, E_{1} \Delta x\right) \leq \frac{\pi E_{1}^{2}}{c_{2}}
$$

b: Now for two given adjacent cells $\Omega_{j}$ and $\Omega_{k}$, we define $C\left(\Omega_{j}, \Omega_{k}\right)$ as the smallest rectangle such that $\Omega_{j} \subset C\left(\Omega_{j}, \Omega_{k}\right)$ and $\Omega_{k} \subset C\left(\Omega_{j}, \Omega_{k}\right)$. That is

$$
C\left(\Omega_{j}, \Omega_{k}\right)=\left[a_{j k}, b_{j k}\right] \times\left[c_{j k}, d_{j k}\right]
$$

with

$$
\begin{aligned}
& a_{j k}=\inf _{(x, y) \in \Omega_{j} \cup \Omega_{k}} x, b_{j k}=\sup _{(x, y) \in \Omega_{j} \cup \Omega_{k}} x, \\
& c_{j k}=\inf _{(x, y) \in \Omega_{j} \cup \Omega_{k}} y, d_{j k}=\sup _{(x, y) \in \Omega_{j} \cup \Omega_{k}} y .
\end{aligned}
$$


It is clear that there exists $E_{3}>0$ such that for all adjacent cells $\left(\Omega_{j}, \Omega_{k}\right)$

$$
\forall\left(M, M^{\prime}\right) \in C\left(\Omega_{j}, \Omega_{k}\right), \quad\left|M-M^{\prime}\right| \leq E_{3} \Delta x
$$

Indeed, since $\exists c>0$ such that the diameter of each cell is smaller than $c \Delta x$, then (70) holds with $E_{3}=2 c$ (the mesh is assumed to be triangular or quadrangular). As a consequence and because of the hypothesis $(63), \exists E_{4}>0$

$$
\operatorname{meas}\left(C\left(\Omega_{j}, \Omega_{k}\right)\right)=\left(b_{j k}-a_{j k}\right) \times\left(c_{j k}-d_{j k}\right) \leq E_{4} \Delta x^{2} .
$$

The constant $E_{4}=E_{4}\left(c_{1}, c_{2}\right)$ is uniform with respect to $j$ and $\Delta x$.

c: Let us now consider $f \in L^{\infty}(\Omega) \cap B V(\Omega)$ and $f_{\Delta x}$ given by the standard $L^{2}$ projection

$$
f_{\Delta x}=\sum_{j \in J}\left(\frac{1}{s_{j}} \int_{\Omega_{j}} f\right) 1_{\Omega_{j}}
$$

We refer to [18] for definitions and properties of the BV norm. In order to simplify the discussion, we assume for a while that $f \in C_{\text {per }}^{\infty}(\Omega)$. Thus the BV semi-norm of $f[18]$ is defined by

$$
\|f\|_{B V}=\int_{\Omega}|\nabla f|
$$

On the other hand, $f_{\Delta x}$ is a discrete profile. So [18]

$$
\begin{aligned}
\left\|f_{\Delta x}\right\|_{B V} & =\sum_{j \in J} \sum_{k \in I^{-}(j) \bigcup I^{0}(j)} l_{j k}\left|\frac{1}{s_{j}} \int_{\Omega_{j}} f-\frac{1}{s_{k}} \int_{\Omega_{k}} f\right| \\
& =\sum_{j \in J} \sum_{k \in I^{-}(j) \bigcup I^{0}(j)} \frac{l_{j k}}{s_{j} s_{k}}\left|\int_{M^{\prime} \in \Omega_{j}} \int_{M \in \Omega_{k}}\left(f(M)-f\left(M^{\prime}\right)\right) \mathrm{d} M \mathrm{~d} M^{\prime}\right| \\
& \leq \sum_{j \in J} \sum_{k \in I^{-}(j) \bigcup I^{0}(j)} \frac{l_{j k}}{s_{j} s_{k}} \int_{M^{\prime} \in \Omega_{j}} \int_{M \in \Omega_{k}}\left|f(M)-f\left(M^{\prime}\right)\right| \mathrm{d} M \mathrm{~d} M^{\prime} \\
& \leq \sum_{j \in J} \sum_{k \in I^{-}(j) \bigcup I^{0}(j)} \frac{l_{j k}}{s_{j} s_{k}} \iint_{M, M^{\prime} \in C\left(\Omega_{j}, \Omega_{k}\right)}\left|f(M)-f\left(M^{\prime}\right)\right| \mathrm{d} M \mathrm{~d} M^{\prime} .
\end{aligned}
$$

For $M=(x, y)$ and $M^{\prime}=\left(x^{\prime}, y^{\prime}\right)$ we bound

$$
\begin{aligned}
\left|f(M)-f\left(M^{\prime}\right)\right| & =\left|\int_{x}^{x^{\prime}} \partial_{x} f(s, y) \mathrm{d} s+\int_{y}^{y^{\prime}} \partial_{y} f\left(x^{\prime}, s\right) \mathrm{d} s\right| \\
& \leq \int_{x}^{x^{\prime}}\left|\partial_{x} f(s, y)\right| \mathrm{d} s+\int_{y}^{y^{\prime}}\left|\partial_{y} f\left(x^{\prime}, s\right)\right| \mathrm{d} s \\
& \leq \int_{x}^{x^{\prime}}\|\nabla f(s, y)\| \mathrm{d} s+\int_{y}^{y^{\prime}}\left\|\nabla f\left(x^{\prime}, s\right)\right\| \mathrm{d} s
\end{aligned}
$$

that is (using notations (69))

$$
\left|f(M)-f\left(M^{\prime}\right)\right| \leq \int_{a_{j k}}^{b_{j k}}\|\nabla f(s, y)\| \mathrm{d} s+\int_{c_{j k}}^{d_{j k}}\left\|\nabla f\left(x^{\prime}, s\right)\right\| \mathrm{d} s .
$$


Next we incorporate this expression in (73) and get

$$
\begin{aligned}
\left\|f_{\Delta x}\right\|_{B V} \leq & \sum_{j \in J} \sum_{k \in I^{-}(j) \cup I^{0}(j)} \frac{l_{j k}}{s_{j} s_{k}} \\
& \times \int_{M^{\prime} \in C\left(\Omega_{j}, \Omega_{k}\right)} \int_{M \in C\left(\Omega_{j}, \Omega_{k}\right)}\left(\int_{a_{j k}}^{b_{j k}}\|\nabla f(s, y)\| \mathrm{d} s\right. \\
& \left.+\int_{c_{j k}}^{d_{j k}}\left\|\nabla f\left(x^{\prime}, s\right)\right\| \mathrm{d} s\right) \mathrm{d} M \mathrm{~d} M^{\prime} \\
= & \sum_{j \in J} \sum_{k \in I^{-}(j) \cup I^{0}(j)} \frac{l_{j k}}{s_{j} s_{k}} \\
& \times \int_{x^{\prime}=a_{j k}}^{b_{j k}} \int_{y^{\prime}=c_{j k}}^{d_{j k}} \int_{x=a_{j k}}^{b_{j k}} \int_{y=c_{j k}}^{d_{j k}}\left(\int_{a_{j k}}^{b_{j k}}\|\nabla f(s, y)\| \mathrm{d} s\right. \\
& \left.+\int_{c_{j k}}^{d_{j k}}\left\|\nabla f\left(x^{\prime}, s\right)\right\| \mathrm{d} s\right) \mathrm{d} y \mathrm{~d} x \mathrm{~d} y^{\prime} \mathrm{d} x^{\prime} .
\end{aligned}
$$

This can be written as

$$
\begin{aligned}
\left\|f_{\Delta x}\right\|_{B V} \leq & \sum_{j \in J} \sum_{k \in I^{-}(j) \cup I^{0}(j)} \frac{l_{j k}}{s_{j} s_{k}} \\
& \times\left(\int_{x^{\prime}=a_{j k}}^{b_{j k}} \int_{y^{\prime}=c_{j k}}^{d_{j k}} \int_{x=a_{j k}}^{b_{j k}}\left(\int_{y=c_{j k}}^{d_{j k}} \int_{a_{j k}}^{b_{j k}}\|\nabla f(s, y)\| \mathrm{d} s \mathrm{~d} y\right) \mathrm{d} x \mathrm{~d} y^{\prime} \mathrm{d} x^{\prime}\right. \\
& \left.+\int_{y^{\prime}=c_{j k}}^{d_{j k}} \int_{x=a_{j k}}^{b_{j k}} \int_{y=c_{j k}}^{d_{j k}}\left(\int_{x^{\prime}=a_{j k}}^{b_{j k}} \int_{c_{j k}}^{d_{j k}}\left\|\nabla f\left(x^{\prime}, s\right)\right\| \mathrm{d} s \mathrm{~d} x^{\prime}\right) \mathrm{d} y \mathrm{~d} x \mathrm{~d} y^{\prime}\right) .
\end{aligned}
$$

Thus we have

$$
\left\|f_{\Delta x}\right\|_{B V} \leq \sum_{j \in J} \sum_{k \in I^{-}(j) \bigcup I^{0}(j)} \frac{l_{j k}}{s_{j} s_{k}}\left(b_{j k}-a_{j k}+d_{j k}-c_{j k}\right)\left(b_{j k}-a_{j k}\right)\left(d_{j k}-c_{j k}\right) \int_{C\left(\Omega_{j}, \Omega_{k}\right)}|\nabla f(M)| \mathrm{d} M,
$$

and, using (71),

$$
\left\|f_{\Delta x}\right\|_{B V} \leq \sum_{j \in J} \sum_{k \in I^{-}(j) \bigcup I^{0}(j)} \frac{l_{j k}}{s_{j} s_{k}}\left(b_{j k}-a_{j k}+d_{j k}-c_{j k}\right) E_{4} \Delta x^{2} \int_{C\left(\Omega_{j}, \Omega_{k}\right)}|\nabla f(M)| \mathrm{d} M .
$$

Due to the uniform regularity of the grid and (70) there exists $C>0$ such that

$$
\frac{l_{j k}}{s_{j} s_{k}}\left(\left|b_{j k}-a_{j k}\right|+\left|d_{j k}-c_{j k}\right|\right) E_{4} \Delta x^{2} \leq C, \forall j, k \text { and } \forall \Delta x \text {. }
$$

Thus we obtain

$$
\left\|f_{\Delta x}\right\|_{B V} \leq E_{5} \sum_{j \in J} \sum_{k \in I^{-}(j) \cup I^{0}(j)} \int_{C\left(\Omega_{j}, \Omega_{k}\right)}|\nabla f(M)| \mathrm{d} M
$$

We rewrite this as

$$
\left\|f_{\Delta x}\right\|_{B V} \leq E_{5} \int_{\Omega}\left|\left(\sum_{j, k \text { such that } M \in C\left(\Omega_{j}, \Omega_{k}\right)} 1\right) \nabla f(M)\right| \mathrm{d} M .
$$

Now we use (70): if $(x, y) \in C\left(\Omega_{j}, \Omega_{k}\right)$ then $\Omega_{j} \subset \mathcal{B}\left(M\right.$, diameter $\left.\left(C\left(\Omega_{j}, \Omega_{k}\right)\right)\right)$. Inequality (70) implies that the number of such cells is bounded

$$
\exists E_{6}>0, \quad \sum_{j, k \text { such that }} 1 \leq E_{6} .
$$


What is important is that $E_{6}$ is independent of the size of the mesh $\Delta x$. So finally

$$
\left\|f_{\Delta x}\right\|_{B V} \leq\left(E_{5} E_{6}\right) \int_{\Omega}|\nabla f(M)| \mathrm{d} M=E_{7}\|f\|_{B V} .
$$

By density of $C^{\infty}$ in $L^{\infty}(\Omega) \cap B V(\Omega)$ and since all constants are independent of the mesh size, it proves the continuity of the $L^{2}$ projection in BV. The constant given here is sufficient for our purposes, but is probably far from being optimal.

\section{REFERENCES}

[1] J.B. Bell, C.N. Dawson and G.R. Shubin, An unsplit higher order Godunov method for scalar conservation laws in multiple dimensions. J. Comp. Phys. 17 (1992) 1-24.

[2] R. Botchorishvili, B. Perthame and A. Vasseur, Schémas d'équilibre pour des lois de conservation scalaires avec des termes sources raides. Report No. 3891, INRIA, France (2000).

[3] C. Chainais-Hillairet, First and second order schemes for a hyperbolic equation: convergence and error estimate, in Finite Volume for Complex Applications Problems and Perspectives, Benkhaldoun and Vilsmeier, Eds., Hermes, Paris (1997) $137-144$.

[4] S. Champier, T. Gallouët and R. Herbin, Convergence of an upstream finite volume scheme for a nonlinear hyperbolic equation on a triangular mesh. Numer. Math. 66 (1993) 139-157.

[5] P.G. Ciarlet, The Finite Element Method for Elliptic Problems. North-Holland Publishing Company, Amsterdam, New York, Oxford (1978).

[6] B. Cockburn, On the continuity in BV of the $L^{2}$-projection into finite element spaces. Math. Comp. 57 (1991) 551-561.

[7] B. Cockburn, F. Coquel and P. Le Floch, An error estimate for finite volume multidimensional conservation laws. Tech. Report No. 285 CMAPX, École Polytechnique, France (1993).

[8] B. Cockburn and C.W. Shu, The Runge-Kutta local projection discontinuous Galerkin finite element method for conservation laws: the multidimensional case. Math. Comp. 54 (1990) 545-581.

[9] P. Collella, Multidimensional upwind methods for hyperbolic conservation laws. J. Comp. Phys. 87 (1990) 171-200.

[10] F. Coquel and P. Le Floch, Convergence of finite difference schemes for conservation laws in several space dimensions: the corrected antidiffusive flux approach. Math. Comp. 57 (1991) 169-210.

[11] R. Dautray and J.-L. Lions, Analyse numérique et calcul numérique pour les sciences et les techniques. Masson, Paris (1984).

[12] H. Deconinck, R. Struijs and G. Bourgeois, Compact advection schemes on unstructured grids, in Computational Fluid Dynamics Lect. Ser. 1993-04, von Karman Institute, Rhode-Saint-Genèse, Belgium (1993).

[13] B. Després and F. Lagoutière, Un schéma non linéaire anti-dissipatif pour l'équation d'advection linéaire. C. R. Acad. Sci., Paris, Sér. I, Math. 328 (1999) 939-944.

[14] B. Després and F. Lagoutière, Contact discontinuity capturing schemes for linear advection and compressible gas dynamics. In preparation.

[15] R.J. DiPerna, Measure value solutions to conservation laws. Arch. Rational Mech. Anal. 88 (1985) 223-270.

[16] F. Dubois and G. Mehlman, A non-parametrized entropy correction for Roe's approximate Riemann solver. Numer. Math. 73 (1996) 169-208.

[17] R. Eymard, T. Gallouët and R. Herbin, Finite volume methods. Tech. Report No. 97-19, LATP, UMR 6632, Marseille, France. To appear in Handbook of Numerical Analysis, P.G. Ciarlet and J.-L. Lions, Eds., Elsevier, Amsterdam.

[18] E. Giusti, Minimal Surfaces and Functions of Bounded Variation. Birkhäuser, Basel (1984).

[19] E. Godlewski and P.-A. Raviart, Numerical approximation of hyperbolic systems of conservation laws, in Applied Mathematical Sciences 118, Springer, New York (1996).

[20] E. Godlewski and P.-A. Raviart, Hyperbolic systems of conservation laws, in Mathématiques 63 Applications 3-4, Ellipses, Paris (1991).

[21] H. Harten, On a class of high resolution total-variation-stable finite-difference schemes. SIAM J. Numer. Anal. 21 (1984) 1-23.

[22] H. Harten, High resolution schemes for hyperbolic conservation laws. J. Comp. Phys. 49 (1983) 357-393.

[23] A. Harten, S. Osher, B. Engquist and S. Chakravarthy, Some results on uniformly High Order Accurate Essentially Nonoscillatory Schemes, in Adv. Numer. Appl. Math., ICASE Report No. 86-18, J.C. South, Jr and M.Y. Hussaini, Eds. (1986) 383-436; J. Appl. Numer. Math. 2 (1986) 347-377.

[24] S.N. Kruzkov, Generalized solutions of the Cauchy problem in the large for non linear equations of first order. Dokl. Akad. Nauk SSSR 187 (1970) 29-32. English translation in Soviet Math. Dokl. 10 (1969).

[25] N.N. Kuznetzov, Finite difference schemes for multidimensional first order quasi-linear equations in classes of discontinuous functions. Probl. Math. Phys. Vych. Mat., Nauka, Moscow (1977) 181-194.

[26] F. Lagoutière, Modélisation mathématique et résolution numérique de problèmes de fluides compressibles à plusieurs constituants. Ph.D. Thesis, Université Pierre et Marie Curie, Paris (2000).

[27] R.J. LeVeque, Numerical Methods for Conservation Laws. ETHZ Zürich, Birkhäuser, Basel (1992). 
[28] R.J. LeVeque, High-resolution conservative algorithms for advection in incompressible flows. SIAM J. Numer. Anal. 33 (1996) $627-665$.

[29] P.-L. Lions, B. Perthame and E. Tadmor, A kinetic formulation of multidimensional scalar conservation laws and related equations. J. Amer. Math. Soc. 7 (1994) 169-191.

[30] S. Osher and E. Tadmor, On the convergence of difference approximations to scalar conservation laws. Math. Comp. 50 (1988) $19-51$.

[31] P.L. Roe, Generalized formulations of TVD Lax-Wendroff schemes. ICASE Report No. 84-53, ICASE, NASA Langley Research Center, Hampton, VA (1984).

[32] P.L. Roe and D. Sidilkover, Optimum positive linear schemes for advection in two and three dimensions. SIAM J. Numer. Anal. 29 (1992) 1542-1568.

[33] P.K. Sweby, High resolution schemes using flux limiters for hyperbolic conservation laws. SIAM J. Num. Anal. 21 (1984) 995-1011.

[34] A. Szepessy, Convergence of a streamline diffusion finite element method for conservation law with boundary conditions. RAIRO Modél. Math. Anal. Numér. 25 (1991) 749-783.

[35] E. F. Toro, Riemann Solvers and Numerical Methods for Fluid Dynamics. Springer-Verlag, Berlin, Heidelberg, New York (1997).

[36] B. Van Leer, Towards the ultimate conservative difference scheme, V. J. Comput. Phys 32 (1979) 101-136.

[37] R.S. Varga, Matrix Iterative Analysis. 2. Printing, in Prentice-Hall Series in Automatic Computation, Prentice-Hall, Inc., Englewood Cliffs, NJ (1963). 